\title{
A turbo SDMA receiver for strongly nonlinearly distorted MC-CDMA signals
}

\author{
Un récepteur turbo SDMA à signaux \\ MC-CDMA soumis à de fortes distorsions \\ non linéaires
}

\author{
Paulo Silva and Rui Dinis*
}

\begin{abstract}
This paper considers the use of space division multiple access (SDMA) techniques for the uplink of multi-carrier code division multiple access (MCCDMA) systems, where the transmitted signals face strong nonlinear distortion effects. The signal transmitted by each mobile terminal (MT) is submitted to a nonlinear operation consisting of a clipping device followed by a frequency-domain filtering operation, so as to reduce the envelope fluctuations and peak-to-mean envelope power ratio (PMEPR) while maintaining the spectral occupation of conventional MC-CDMA signals. At the base station, an iterative receiver employing multiple antennas jointly performs turbo multi-user detection and the estimation and cancellation of nonlinear distortion effects. Performance results show that the proposed receiver structures can achieve good performances that are very close to those obtained with linear transmitters, even for high system load and/or when a very low-PMEPR MC-CDMA transmission is intended for each MT.

Cet article envisage l'utilisation des techniques de division spatiale à accès multiple ou SDMA en voie montante pour les systèmes à accès multiple par répartition en codes multiporteuses ou MC-CDMA où le signal transmis est soumis à de fortes distorsions non linéaires. Le signal transmis par chaque terminal mobile est soumis à une operation non linéaire consistant d'un dispositif de coupure suivi d'une opération de filtrage au niveau du domaine de fréquence de manière à réduire les fluctuations de l'enveloppe ainsi que le rapport pic-moyenne de la puissance de l'enveloppe (PMEPR), tout en maintenant l'occupation spectrale des signaux MC-CDMA conventionnels. À la station de base, un récepteur itératif utilisant simultanément plusieurs antennes fait la détection multi-utilisateur ainsi que l'estimation et l'annulation des effets de distortion non linéaires. Les résultats montrent que les structures de récepteur proposées conduisent à de bonnes performances très proches de celles obtenues avec les transmetteurs linéaires, et cela même pour les systèmes à charge élevée et/ou quand une transmission MC-CDMA à tres bas PMEPR est prévue pour chaque station de base.
\end{abstract}

Keywords: multi-carrier code division multiple access (MC-CDMA); multi-user detection; nonlinear effects; space division multiple access (SDMA); turbo equalization

\section{Introduction}

Space division multiple access (SDMA) techniques employ multiple antennas to substantially increase the system capacity [?]. In this paper we consider the use of SDMA techniques for the uplink of multicarrier code division multiple access (MC-CDMA) systems. As in other multi-carrier schemes, MC-CDMA signals have strong envelope fluctuations and high peak-to-mean envelope power ratio (PMEPR) values, leading to amplification difficulties. For this reason, it is desirable to reduce the envelope fluctuations of the transmitted signals. This is particularly important for the uplink transmission, since an efficient, low-cost power amplification is desirable at the mobile terminal (MT). Several techniques have been recommended to reduce the envelope fluctuations of multi-carrier signals (see [?] and references therein). One promising approach is to employ clipping techniques combined with frequency-domain filtering so as to reduce the envelope fluctuations of the transmitted signals while maintaining the spectral occupation of conventional schemes [?]. However, the nonlinear distortion effects can be severe when a low-PMEPR transmission is intended [?]-[?].

As in other CDMA schemes, since the transmission over timedispersive channels destroys the orthogonality between users, a frequency-domain equalizer (FDE) is required before the despread-

* Paulo Silva is with ISR-IST/EST, University of Algarve, Campus da Penha, 8005-139 Faro, Algarve, Portugal. E-mail: psilva@ualg.pt. Rui Dinis is with ISR-IST, Technical University of Lisbon, Av. Rovisco Pais, Torre Norte, Piso 7, 1049-001 Lisbon, Lisbon, Portugal. E-mail: rdinis@ ist.utl.pt. ing operation [?]. To avoid significant noise enhancement for channels with deep in-band notches, the FDE is usually optimized under a minimum mean-square error (MMSE) criterion [?]. However, since an MMSE FDE does not perform an ideal channel inversion, we are not able to fully orthogonalize the different spreading codes of an MCCDMA signal. ${ }^{1}$ This means that we can have severe interference levels, especially for fully loaded systems and/or when different powers are assigned to different spreading codes. To improve performance, several turbo multi-user detection (MUD) receivers have been proposed for conventional CDMA systems [?]-[?], as well as MC-CDMA [?]. A promising technique for MC-CDMA with nonlinear transmitters was proposed in [?], where nonlinear distortion effects are iteratively estimated and compensated. However, for low SNR, the error decisions might lead to error propagation effects, since errors in the estimation of nonlinear distortion effects can preclude an efficient cancellation. This is particularly serious for high system load and/or when no space diversity is used [?], and it is especially important when the spreading factor is small and/or if we decrease the clipping level to further reduce the PMEPR of the transmitted signals.

To reduce error propagation effects in the typical region of operation, we use channel decoder outputs in the feedback loop in a turbo-like fashion (a similar approach has been proposed for orthogonal frequency-division multiplexing (OFDM) schemes [?]). We de-

\footnotetext{
${ }^{1}$ An MMSE FDE might also lead to noise correlations, creating unwanted dependencies between the decisions made for each data symbol associated with a given spreading code. This is usually not a problem in coded systems, provided that a suitable interleaving is employed between the channel encoder and the symbol mapper.
} 


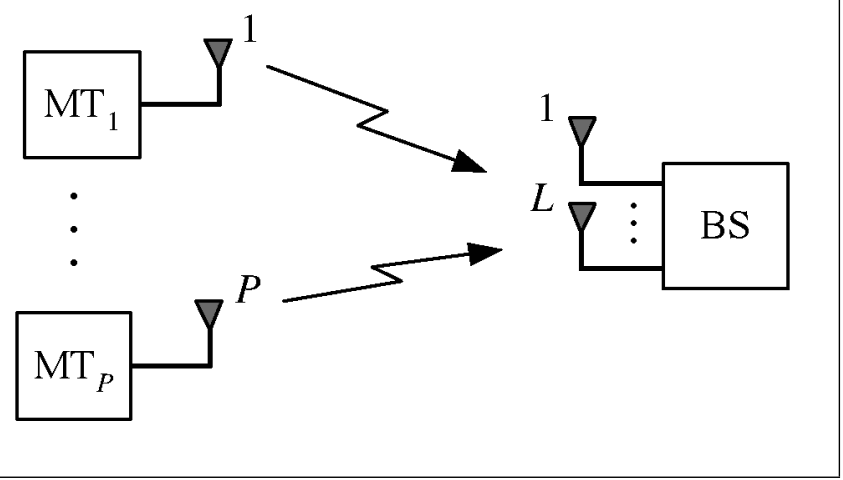

Figure 1: System characterization.

fine an iterative receiver that jointly performs turbo MUD as well as estimation and cancellation of nonlinear distortion effects, taking into account the frequency distribution of the distortion that is inherent to the transmitted signals.

This paper is organized as follows: The system characterization considered here is described in Section II. In Section III, we describe the iterative receivers proposed in this paper. Section IV presents a set of performance results, and Section $\mathrm{V}$ is concerned with the conclusions of the paper.

\section{System characterization}

We consider the uplink transmission of MC-CDMA signals employing frequency-domain spreading. We have an SDMA architecture depicted in Fig. 1, corresponding to a multiple-input, multiple-output (MIMO) system with $P$ users (MTs) transmitting independent data blocks and $L$ receive antennas at the base station (BS). It is assumed that each MT has a single transmit antenna. The coded bits are interleaved and mapped, leading to the block of data symbols to be transmitted by the $p$-th MT $\left\{A_{k, p} ; k=0,1, \ldots, M-1\right\}$, where $M$ is the number of data symbols for that MT. The frequency-domain block to be transmitted by the $p$-th MT is $\left\{S_{k, p} ; k=0,1, \ldots, N-1\right\}$, where $N=K M$, with $K$ denoting the spreading factor. The frequencydomain symbols are given by $S_{k, p}=\xi_{p} C_{k, p} A_{k \bmod M, p}(x \bmod y$ is the remainder of the division of $x$ by $y$ ), where $\xi_{p}$ is an appropriate weighting coefficient that accounts for the propagation losses and $\left\{C_{k, p} ; k=0,1, \ldots, N-1\right\}$ is the corresponding spreading sequence $^{2}$ (a pseudo-random spreading is assumed, with $C_{k, p}$ belonging to a QPSK constellation; without loss of generality, it is assumed that $\left|C_{k, p}\right|=1$ ). The transmitter structure depicted in Fig. 2 is based on the nonlinear signal processing schemes proposed in [?] for reducing the PMEPR of OFDM signals. Within that transmitter, $N^{\prime}-N$ zeros are added to the original frequency-domain block (i.e., $N^{\prime}-N$ idle subcarriers), followed by an inverse discrete Fourier transform (IDFT) operation so as to generate a sampled version of the time-domain MCCDMA signal with an oversampling factor $M_{T x}=N^{\prime} / N$. Each timedomain sample is submitted to a nonlinear device so as to reduce the envelope fluctuations on the transmitted signal. In this paper, we assume that the nonlinear device is an ideal envelope clipping with clipping level $s_{M}$, i.e., the output samples are

$$
s_{n, p}^{c}= \begin{cases}s_{n, p}^{\prime}, & s_{n, p}^{\prime} \leq s_{M}, \\ s_{M}, & s_{n, p}^{\prime}>s_{M},\end{cases}
$$

with $\left\{s_{n, p}^{\prime} ; n=0,1, \ldots, N^{\prime}-1\right\}$ denoting the input samples. After a discrete Fourier transform (DFT) operation, the clipped signal is

\footnotetext{
${ }^{2}$ This corresponds to uniformly spreading the chips associated with a given symbol within the transmission band, i.e., to employing a rectangular interleaver with dimensions $K \times M$.
}

submitted to a frequency-domain filtering procedure through the set of multiplying coefficients $G_{k}, k=0,1, \ldots, N^{\prime}-1$, in order to reduce the out-of-band radiation levels inherent to the nonlinear operation.

Using Price's theorem [?], it is shown in [?] that the frequencydomain block to be transmitted by the $p$-th $\operatorname{MT}\left\{S_{k, p}^{T x}=\right.$ $\left.S_{k, p}^{C} G_{k} ; k=0,1, \ldots, N^{\prime}-1\right\}$ can be decomposed into the sum of two uncorrelated components: a useful one, proportional to $\left\{S_{k, p} ; k=0,1, \ldots, N-1\right\}$, and a nonlinear self-interference one, i.e., $S_{k, p}^{T x}=\alpha_{p} S_{k, p} G_{k}+D_{k, p} G_{k}$, where $\alpha_{p}$ is a scalar factor, defined in [?] and [?]; $G_{k}, k=0,1, \ldots, N^{\prime}-1$, are the frequency-domain filtering coefficients required to reduce the out-of-band radiation levels inherent to the nonlinear operation; and $\left\{D_{k, p} ; k=0,1, \ldots, N^{\prime}-1\right\}$ is the frequency-domain block of nonlinear self-interference components associated with the $p$-th MT. Unless otherwise stated, we will assume that $G_{k}=1$ for the $N$ in-band subcarriers and 0 for the $N^{\prime}-N$ out-of-band subcarriers, i.e., the spectral occupation of the transmitted signal is similar to the spectral occupation of conventional MC-CDMA signals. In this case, $S_{k, p}^{T x}=\alpha_{p} S_{k, p}+D_{k, p}$ for the $N$ in-band subcarriers, and 0 otherwise. It can be shown that $D_{k, p}$ is approximately Gaussian-distributed with zero mean; moreover, $E\left[D_{k, p} D_{k^{\prime}, p}^{*}\right]$ can be computed analytically, as described in [?] and [?].

\section{Receiver structure}

\section{III.A Linear transmitters}

As usual, it is assumed that the length of the cyclic prefix (CP) is higher than the length of the overall channel impulse response. The received time-domain block associated with the $l$-th diversity branch, after the samples associated with the CP are discarded, is $\left\{y_{n}^{(l)} ; n=\right.$ $0,1, \ldots, N-1\}$. The corresponding frequency-domain block (i.e., the length- $N$ DFT of the block $\left.\left\{y_{n}^{(l)} ; n=0,1, \ldots, N-1\right\}\right)$ is $\left\{Y_{k}^{(l)} ; k=0,1, \ldots, N-1\right\}$.

Let us consider first a linear transmitter. In this case, the frequencydomain block transmitted by the $p$-th MT is $\left\{S_{k, p}^{T x}=S_{k, p} ; k=\right.$ $\left.0,1, \ldots, N^{\prime}-1\right\}$ and

$$
\begin{aligned}
Y_{k}^{(l)} & =\sum_{p=1}^{P} S_{k, p} H_{k, p}^{\mathrm{Ch}(l)}+N_{k}^{(l)} \\
& =\sum_{p=1}^{P} A_{k \bmod M, p} C_{k, p} \xi_{p} H_{k, p}^{\mathrm{Ch}(l)}+N_{k}^{(l)} \\
& =\sum_{p=1}^{P} A_{k \bmod M, p} H_{k, p}^{(l)}+N_{k}^{(l)},
\end{aligned}
$$

with $H_{k, p}^{\mathrm{Ch}(l)}$ denoting the channel frequency response between the $p$-th MT and the $l$-th diversity branch at the $k$-th subcarrier; $N_{k}^{(l)}$ denoting the corresponding channel noise; and $H_{k, p}^{(l)}$ representing $\xi_{p} H_{k, p}^{\mathrm{Ch}(l)} C_{k, p}$. To detect the $k$-th symbol of the $p$-th MT, we will use the set of subcarriers $\Psi_{k}=\{k, k+M, \ldots, k+(K-1) M\}$.

By defining $\mathbf{Y}(k)=\left[\mathbf{Y}^{(1)}(k) \cdots \mathbf{Y}^{(L)}(k)\right]^{T}$, with $\mathbf{Y}^{(l)}(k)$ denoting the column vector with the received samples associated with the set of frequencies $\Psi_{k}$, for the $l$-th antenna, and $\mathbf{A}(k)=$ $\left[A_{k \bmod M, 1} \ldots A_{k \bmod M, P}\right]^{T}$, we have

$$
\mathbf{Y}(k)=\mathbf{H}^{T}(k) \mathbf{A}(k)+\mathbf{N}(k),
$$

where $(\cdot)^{T}$ denotes the transpose matrix and $\mathbf{N}(k)=$ $\left[\mathbf{N}^{(1)}(k) \cdots \mathbf{N}^{(L)}(k)\right]^{T}$, with $\mathbf{N}^{(l)}(k)$ denoting the column vector with the noise samples associated with the set of frequencies $\Psi_{k}$, for the $l$-th antenna. In (??), $\mathbf{H}(k)$ is the overall channel matrix associated with $\mathbf{A}(k)$, i.e., $\mathbf{H}(k)=\left[\mathbf{H}^{(1)}(k) \cdots \mathbf{H}^{(L)}(k)\right]$, with 